\title{
Radiation performance of new semiconductor power devices for the LHC experiment upgrades
}

C. Abbate ${ }^{(g, d)}$, M. Alderighi $i^{(f, a)}$, S. Baccaro ${ }^{(e, d)}$, G. Busatto ${ }^{(g, d)}$, M. Citterio $^{(a)}, \mathbf{P}$. Cova $^{(j, c)}$, N. Delmonte ${ }^{(j, c)}$, V. De Luca ${ }^{(g, d)}$, S. Fiore ${ }^{(e, c)}$, S. Gerardin ${ }^{(i, b, ")}$, E. Ghisolfi ${ }^{k}$, F. Giuliani $^{(h)}$, F. lannuzzo ${ }^{(g, d)}$, A. Lanza ${ }^{(c)}$, S. Latorre ${ }^{(a)}$, M. Lazzaroni ${ }^{(h, a)}$, G.

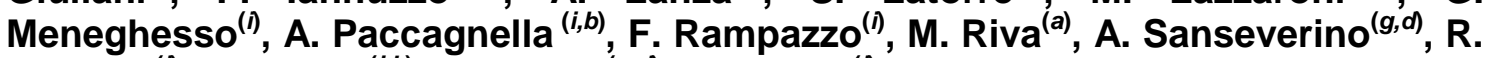
Silvestri ${ }^{(i)}$, G. Spiazzi $i^{(i, b)}$, F. Velardi ${ }^{(g, d)}$, E. Zanoni ${ }^{(i)}$

${ }^{a}$ INFN Milano, via Celoria 16, 20133 Milano (Italy)

${ }^{b}$ INFN Padova, via Marzolo 8, 35131 Padova (Italy)

${ }^{c}$ INFN Pavia, via Bassi 6, 27100 Pavia (Italy)

${ }^{d}$ INFN Roma, p.le Aldo Moro 2, 00185 Roma (Italy)

${ }^{e}$ ENEA UTTMAT, via Anguillarese 301, 00123 Santa Maria di Galeria, Roma (Italy)

${ }^{f}$ INAF-IASF, via Bassini 15, 20133 Milano (Italy)

${ }^{g}$ Dipartimento di Ingegneria Elettrica e dell'Informazione, University of Cassino and Southern Lazio,via Di Biasio 43, 03043 Cassino (Italy)

${ }^{h}$ Dipartimento di Fisica, Università degli Studi di Milano, via Celoria 16, 20133 Milano (Italy)

${ }^{i}$ Dept. of Information Engineering, University of Padova, via Gradenigo 6/A, 35131 Padova (Italy)

${ }^{j}$ Dipartimento di Ingegneria dell'Informazione, University of Parma, viale G.P. Usberti 181/A, 43124

Parma (Italy)

${ }^{k}$ FN S.p.A., SS35bis dei Giovi km 15, 15062 Bosco Marengo (Italy)

*Corresponding author

E-mail: simone.gerardin@dei.unipd.it

$\mathrm{GaN}$ and $\mathrm{SiC}$ power devices were extensively tested under different types of radiation, in the framework of the APOLLO R\&D collaboration, aiming to use these new technologies for designing power supplies for the future LHC experiments upgrades.

$\mathrm{SiC}$ power MOSFETs were irradiated with $\gamma$-rays, neutrons, and heavy ions (Iodine, Bromine) at different energies $(20 \mathrm{MeV}-550 \mathrm{MeV})$. They showed very good performances in terms of Total Ionizing Dose (TID) sensitivity, but exhibited a quite poor Safe Operating Area (SOA) with respect to Single Event Effects (SEEs).

Enhancement-mode GaN transistors manufactured by EPC, with blocking voltage ranging from 40V to $200 \mathrm{~V}$, were irradiated with $\gamma$-rays, heavy ions (Iodine, Bromine), and low energy protons. They showed a very good SOA toward SEE. After the irradiation with $3-\mathrm{MeV}$ protons at the highest fluence $\left(4 \cdot 10^{14}\right.$ $\mathrm{p} / \mathrm{cm}^{2}$ ), the devices exhibited an increase of up to one order of magnitude in gate leakage, almost $1 \mathrm{~V}$ of threshold voltage reduction, degradation of the subthreshold slope, and drop in transconductance. The reduction in threshold is in contrast with the increase normally observed in GaN devices irradiated with protons, and is likely due to radiation effects in the layers introduced to engineer the positive threshold voltage.

11th International Conference on Large Scale Applications and Radiation Hardness of Semiconductor Detectors

July 3-5, 2013 Florence, Italy 


\section{Introduction}

Gallium Nitride and Silicon Carbide-based power devices offer interesting advantages over standard silicon devices, also from a radiation hardness standpoint.

GaN-based HEMTs (High Electron Mobility Transistors) are very attractive thanks to the outstanding properties of Gallium Nitride, a wide bandgap III-V semiconductor material. GaN electronic devices promise to deliver more output power and achieve higher efficiency than transistors made from either silicon or gallium arsenide, due to the wide bandgap (3.4 eV) which enables higher saturated electron velocities and higher breakdown fields.

In addition, bulk GaN material is radiation hard [1]. In fact, the minimum energy to displace $\mathrm{GaN}$ atoms is larger than in $\mathrm{Si}$ and $\mathrm{GaAs}$, and close to that of $\mathrm{SiC}$. Also Total Ionizing Dose (TID) tolerance is outstanding. The devices exploit the properties of heterostructures, and the channel is formed through band engineering, without the use of dielectric layers underneath the gate, which are Achilles' heel of Si-based power MOSFETs, as far as TID effects are concerned.

At the beginning of the 2000's, the total dose sensitivity of GaN HEMTs was investigated, concluding that the degradation was not appreciable up to very high doses (hundreds of Mrad). The vast majority of the literature on radiation effects in GaN HEMTs deals with displacement damage after proton irradiation. Data show that these GaN-based HEMTs, with few exceptions, are able to withstand proton fluences in excess of $10^{12} \mathrm{p} / \mathrm{cm}^{2}$ with only minor degradation.

Silicon Carbide is a wide bandgap III-V semiconductor material too and enables higher breakdown fields like $\mathrm{GaN}$. SiC permits to deliver much larger output power than $\mathrm{GaN}$, because in $\mathrm{SiC}$ devices the output current flows vertically contrarily to GaN where the current flows along a surface. This characteristic permits to achieve high voltage power devices (Schottky power diodes, MOSFETs rated up to $1.7 \mathrm{kV}$ [2], and bipolar IGBTs rated up to $15 \mathrm{kV}$ [3]) with current capabilities comparable or larger than lower voltage Silicon and GaN devices.

$\mathrm{SiC}$ devices have an epitaxial layer much thinner and more doped with respect to silicon devices with comparable blocking voltages so in principle they have a lower sensitive volume to the TID and Single Event Effect (SEE) involving the semiconductor part of the device.

Some works have been dedicated to study SEEs in SiC power diodes [4], power Schottky diodes [5] and MESFETs [6]. TID effects have been studied on SiC BJTs [7] and power MOSFETs [8].

The objective of the present work is to present the results of an extended characterization of $\mathrm{GaN}$ and $\mathrm{SiC}$ power devices. These devices were tested under different types of radiation, in the framework of the APOLLO R\&D collaboration, aiming to verify the possibility of using these new technologies for designing power supplies for the future LHC experiments upgrades. 


\section{Devices and Experiments}

The irradiation tests were executed at the following irradiation facilities:

- $\gamma$-rays: at CALLIOPE, ENEA-UTTMAT, Casaccia, Rome, Italy;

- Neutrons: at TAPIRO, ENEA, Casaccia, Rome, Italy;

- Low energy Protons: at CN accelerator of Laboratori Nazionali di Legnaro (LNL), INFN, Padua, Italy;

- Heavy ions: at TANDEM-XTU and TANDEM-ALPI accelerators of the Laboratori Nazionali di Legnaro (LNL), INFN, Padua, Italy.

\section{Sic Power MOSFET}

The first set of tested devices include power MOSFET manufactured by CREE on SiC rated at $1200 \mathrm{~V} 24 \mathrm{~A}$.

The time durations of the $\gamma$-ray irradiations were set to achieve increasing doses $(0.57,1.1$, $1.7,5.2$, and $10.8 \mathrm{kGy}$ ) using a dose rate of $23.8 \mathrm{~Gy} / \mathrm{h}$. After the irradiation the samples were subjected to one week $100^{\circ} \mathrm{C}$ thermal annealing.

Neutron irradiations were performed with increasing doses: $0.39,0.68,0.52,2.6710^{12}$ $\mathrm{n} / \mathrm{cm}^{2} 1 \mathrm{MeV}$ equivalent (Si) with a dose rate of $3.410^{11}$ neutrons $/ \mathrm{cm}^{2} / \mathrm{h} 1 \mathrm{MeV}$ equivalent (Si).

During $\gamma$-ray irradiations the Devices Under Test (DUTs), were statically biased with $\mathrm{V}_{\mathrm{GS}}=16 \mathrm{~V}$ (corresponding to $80 \%$ of the maximum gate voltage) and $\mathrm{V}_{\mathrm{DS}}=0 \mathrm{~V}$.

The neutron irradiations were performed on DUTs biased with $\mathrm{V}_{\mathrm{DS}}=960 \mathrm{~V}$ (80\%di 1200) and $\mathrm{V}_{\mathrm{GS}}=-3 \mathrm{~V}$.

A group of $\mathrm{SiC}$ power MOSFETs was irradiated with ${ }^{79} \mathrm{Br}$ at energies ranging between 20 and $240 \mathrm{MeV}$ (obtained with TANDEM-XTU) and $550 \mathrm{MeV}$ (TANDEM-ALPI). During the irradiations DUTs were biased with $\mathrm{V}_{\mathrm{GS}}=0 \mathrm{~V}$ and $\mathrm{V}_{\mathrm{DS}}$ increasing up to the failure voltage. The circuit and the test procedure used for heavy ion irradiations, omitted here for brevity, can be found in [9].

\section{GaN HEMT}

The second set of tested devices consists of two types of enhancement-mode GaN High Electron Mobility Transistors manufactured by Efficient Power Conversion (EPC). They have a blocking voltage of 40 or $200 \mathrm{~V}$ and a maximum continuous $\mathrm{I}_{\mathrm{DS}}$ of 12 or $33 \mathrm{~A}$, and a maximum pulsed current of 60 or $150 \mathrm{~A}$.

Some GaN HEMTs were irradiated with $\gamma$-rays in the same irradiation campaign of $\mathrm{SiC}$ power MOSFETs at increasing doses $(0.57,1.1,1.7,5.2$, and $10.8 \mathrm{kGy})$ with a dose rate of 23.8 $\mathrm{Gy} / \mathrm{h}$ and then were subjected to one week $100^{\circ} \mathrm{C}$ thermal annealing. During the irradiation, DUTs were biased with $\mathrm{V}_{\mathrm{GS}}=5 \mathrm{~V}$ (corresponding to $80 \%$ of the maximum gate voltage) and $\mathrm{V}_{\mathrm{DS}}=0 \mathrm{~V}$.

A second group of samples was irradiated with ${ }^{79} \mathrm{Br}$ at $240 \mathrm{MeV}$ and with ${ }^{125} \mathrm{I}$ at $300 \mathrm{MeV}$, both obtained with TANDEM-XTU.

Furthermore, a third group of devices was irradiated with low-energy protons at the $\mathrm{CN}$ accelerator of the Legnaro National Laboratories (LNL), with a fluence up to $4 \cdot 10^{14} \mathrm{p} / \mathrm{cm}^{2}$. The devices were left unbiased during the exposure. DC parameters have been measured before and after $3-\mathrm{MeV}$ proton irradiation. It is worth to note that the energy of these protons is too low to 
give rise to secondary particles, so that no indirect SEEs are generated during the exposures. Instead degradation mainly comes from displacement damage.

\section{Results and Discussion}

\subsection{SiC MOSFETs}

The effects of $\gamma$-ray irradiations on the characteristics of SiC MOSFETs are summarized in Figs. 1-3 where threshold voltage, $\mathrm{R}_{\mathrm{on}}$, and $\mathrm{I}_{\mathrm{GSS}}$ measured after $\gamma$-ray irradiations are reported as a function of the dose absorbed during the irradiation.

Small changes are observed in $\mathrm{R}_{\mathrm{on}}$ and $\mathrm{I}_{\mathrm{GSs}}$ up to the highest dose. A significant reduction of the threshold voltage (Fig. 1) is observed at increasing doses down to $-0.25 \mathrm{~V}$ for $10.8 \mathrm{kGy}$. This significant change does not affect the use of this device in power supplies for the ATLAS upgrade, namely $10 \mathrm{kGy}$. In fact, to guarantee that the device is off a suitatable driver able to apply a negative voltage (i.e. $-3 \mathrm{~V}$ ) will be required.

SiC power MOSFETs are very robust with respect to the sensitivity to Single Event Effect induced by neutrons. In fact, no Single Event Burnout, SEB, or Single Event Gate Rupture, SEGR, was detected up to $2.710^{12} \mathrm{n} / \mathrm{cm}^{2} 1 \mathrm{MeV}$ equivalent ( $\mathrm{Si}$ ) which is about $20 \%$ of the maximum expected dose of ATLAS upgrade, namely $1.610^{13} \mathrm{n} / \mathrm{cm}^{2} 1 \mathrm{MeV}$ equivalent (Si). All the characteristics of the neutron irradiated SiC power MOSFET (threshold voltage, $\mathrm{R}_{\mathrm{on}}$, and $\mathrm{I}_{\mathrm{GSS}}$ ) had practically no changes as indicated in Fig. 4 where the threshold voltage measured after irradiations with increasing dose is reported. The measures of $R_{\mathrm{on}}$ and $\mathrm{I}_{\mathrm{GSS}}$ after irradiations are not reported here for brevity.

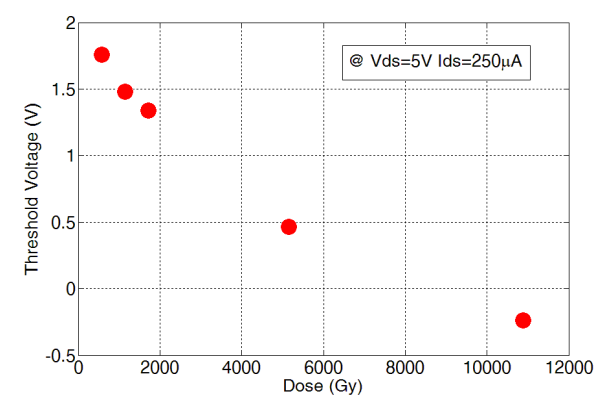

Fig.1. Threshold voltage of SiC MOSFET measured after $\gamma$-ray irradiations as a function of the absorbed dose.

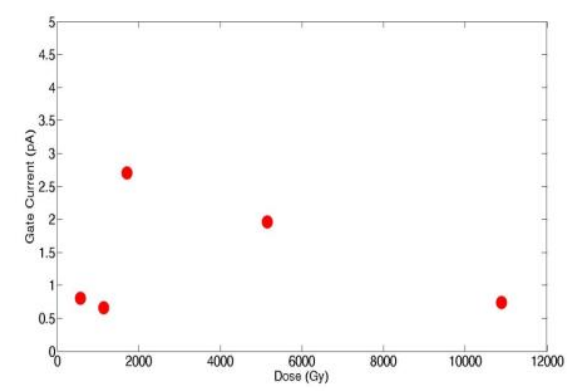

Fig.3. $\mathrm{I}_{\mathrm{GSS}}$ leakage current of SiC MOSFET measured after $\gamma$-ray irradiations as a function of the absorbed dose.

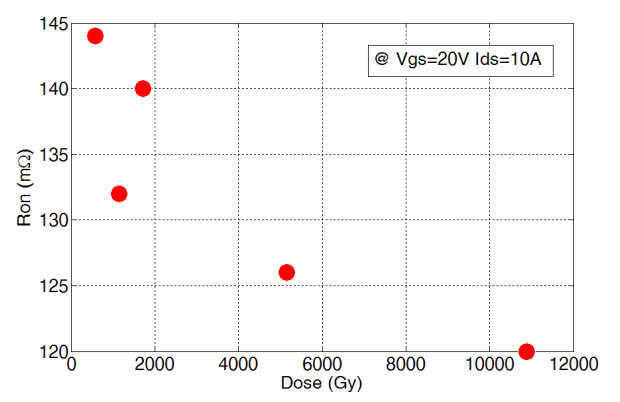

Fig.2. $\mathrm{R}_{\mathrm{on}}$ of SiC MOSFET measured after $\gamma$-ray irradiations as a function of the absorbed dose.

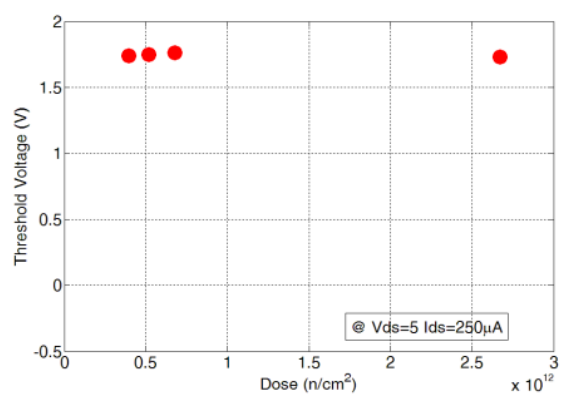

Fig.4. Threshold voltage of SiC MOSFET measured after neutron irradiations as a function of the absorbed dose. 


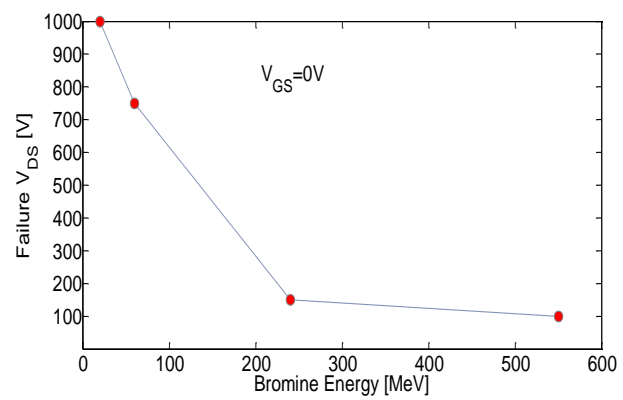

Fig.5. Failure voltage during ${ }^{79} \mathrm{Br}$ irradiations as a function of the beam energy.

SiC power MOSFETs have revealed a significant sensitivity to Single Event Effect induced by heavy ions. In Fig. 5 the failure voltage at which SEB/SEGR were detected during the irradiation with ${ }^{79} \mathrm{Br}$ irradiation at increasing energy is reported.

Fig. 5 indicates that the voltage at which the device can be safely used drastically reduces down to $100 \mathrm{~V}$ if the devices have a finite probability of being impacted by a highly energetic heavy ion. This limitation does not affect the use of SiC power MOSFET in the ATLAS upgrade for which this probability is very low.

\subsection{GaN HEMTs}

A first group of GaN HEMTs rated at $40 \mathrm{~V}$ and $200 \mathrm{~V}$ were irradiated with ${ }^{79} \mathrm{Br}$ at $240 \mathrm{MeV}$ and ${ }^{125} \mathrm{I}$ at $300 \mathrm{MeV}$ obtained with TANDEM-XTU. No SEB/SEGR was detected up to the maximum rated voltage both at the drain and gate sides. So we can conclude that these devices have a full Safe Operating Area with respect to SEE. After this result we strongly believe that their sensitivity to SEE induced by neutrons is very good even if the devices have not been tested yet.

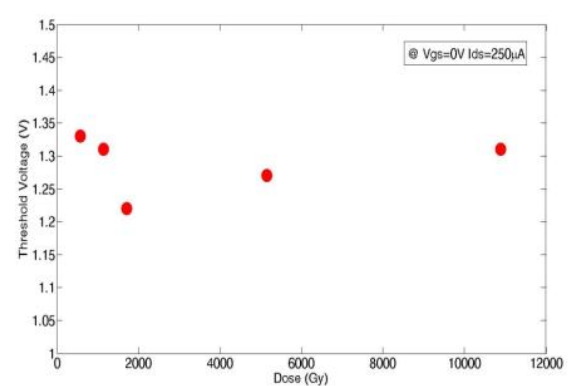

Fig.6. Threshold voltage of GaN HEMT measured after $\gamma$-ray irradiations as a function of the absorbed dose.

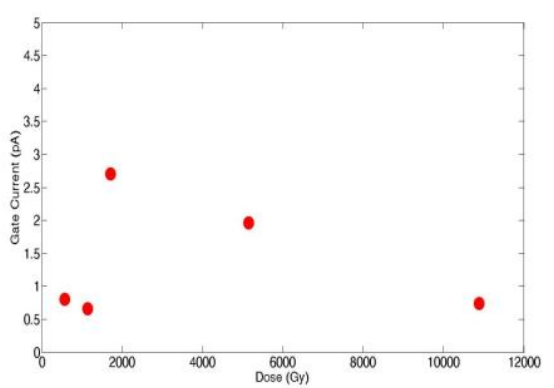

Fig.8. I ISS leakage current of GaN HEMT measured after $\gamma$-ray irradiations as a function of the absorbed dose.

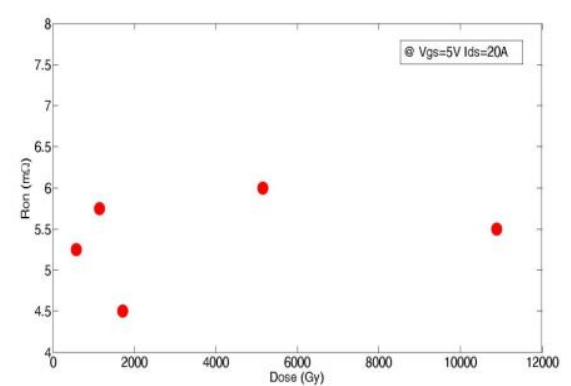

Fig.7. $\mathrm{R}_{\text {on }}$ of GaN HEMT measured after $\gamma$-ray irradiations as a function of the absorbed dose..

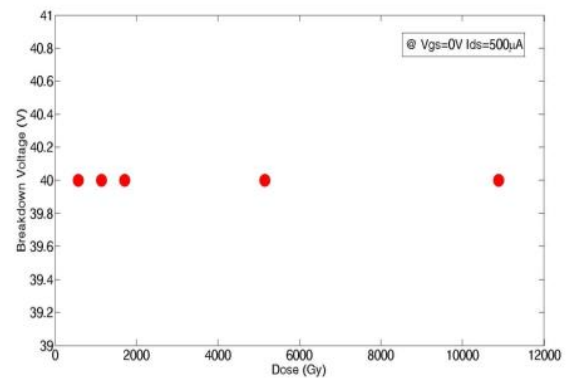

Fig.9. Breakdown Voltage of GaN HEMT measured after $\gamma$-ray irradiations as a function of the absorbed dose. 


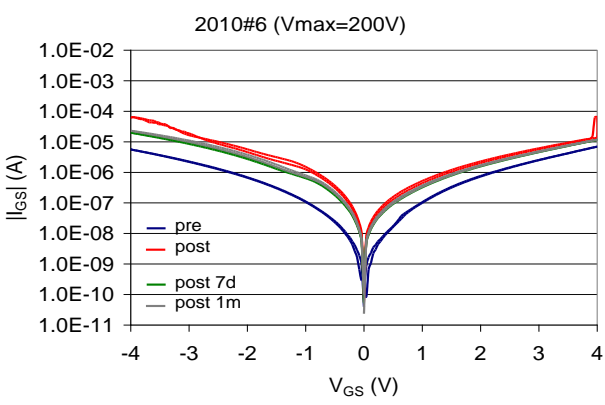

Fig.9. Gate current of an enhancment-mode GaN HEMT irradiated with $10^{14}, 3-\mathrm{MeV}_{\mathrm{p}} / \mathrm{cm}^{2}$.

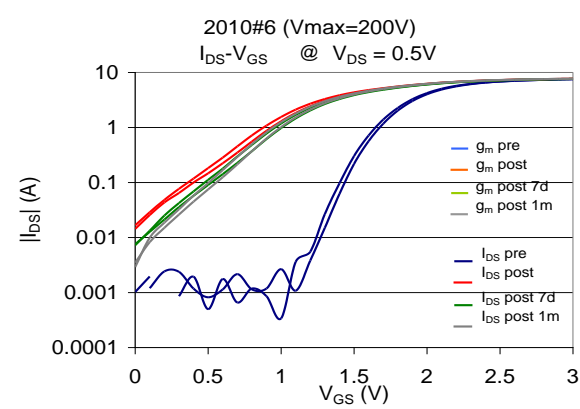

Fig.10. Drain current (log scale) of an enhancment-mode GaN HEMT irradiated with $10^{14}, 3-\mathrm{MeV}$ p/cm ${ }^{2}$

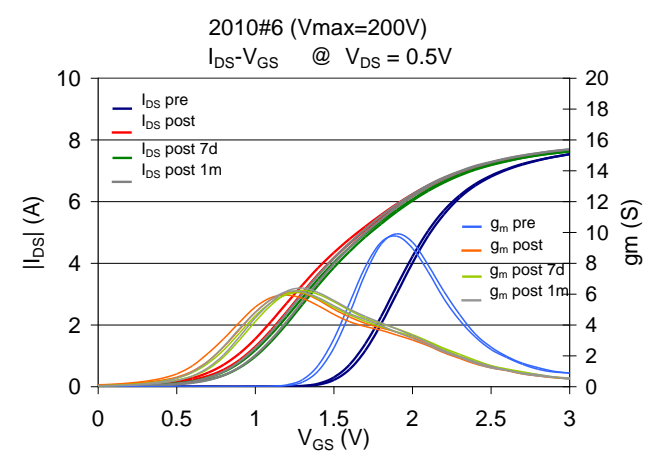

Fig.11. Transconductance and drain current (linear scale) of an enhancment-mode GaN HEMT irradiated with $10^{14}$ $3-\mathrm{MeV} \mathrm{p} / \mathrm{cm}^{2}$.

GaN HEMTs have revealed a very good tolerance to total dose radiation effects. In Figs. 6-9, we report threshold voltage, $\mathrm{R}_{\mathrm{on}}, \mathrm{I}_{\mathrm{GSS}}$, and breakdown voltage measured on $40 \mathrm{~V} \mathrm{GaN}$ HEMT after $\gamma$-ray irradiations as a function of the dose absorbed during the irradiation. The variation of all above parameters are negligible up to the maximum dose of $10.8 \mathrm{kGy}$.

GaN HEMTs were irradiated with $3 \mathrm{MeV}$ protons up to a fluence of $4 \cdot 10^{14} \mathrm{p} / \mathrm{cm}^{2}$. After this irradiation, observed effects include:।

- increase in gate current

- threshold voltage reduction

- transconductance drop.

As shown in Fig. 9, the increase in gate current, up to one order of magnitude, is visible at all voltages, but it is more pronounced for negative voltages. It partially anneals during room temperature storage.

Fig. 10 shows the transfer characteristics in logarithmic scale: an unexpected decrease in threshold voltage of about $1 \mathrm{~V}$, with a degraded substreshold slope, is visible in the post-rad curves. There is modest recovery during room temperature annealing.

Fig. 11 shows that the peak transconductance drops more than $30 \%$ after exposure to 4 $\cdot 10^{14} \mathrm{p} / \mathrm{cm}^{2}$. The same figure also shows that the maximum drain current is almost unchanged after irradiation. That is because the threshold voltage reduction offsets the transconductance degradation, resulting in unchanged drive current at the maximum tested gate voltage.

Protons induce both ionization and displacement, so that the observed degradation can result from the superimposition of displacement effects and ionization effects. The maximum total ionizing dose delivered to the samples is almost $5 \mathrm{MGy}(\mathrm{Si})$. However, as shown before, 
exposures to gamma rays should have minimal impact on the characteristics, meaning that the underlying degradation mechanism is displacement. Yet, much lower ionizing doses were delivered with gamma irradiations $(10 \mathrm{kGy}(\mathrm{Si}))$ than with low-energy protons, so no conclusive evidence is available.

In the literature, a decrease in drain current is typically reported on depletion-mode devices irradiated with protons. For instance [10] presents a compilation of several test data over the course of ten years for GaN HEMTs. All devices show decreases in drain current in contrast with the behavior of these enhancement-mode samples. It is not clear if this difference is due to unknown process steps or introduction of other layers.

We also investigated the damage dependence on proton fluence and blocking voltage: the results show a small dependence of degradation on blocking voltage (40V vs $200 \mathrm{~V}$ ) and a small dependence also on proton fluence, accompanied by considerable sample-to-sample variability.

\section{Conclusions}

SiC power MOSFET have revealed very good tolerance to $\gamma$-rays and neutrons irradiation but they have a low tolerance to SEE induced by high energy heavy ions $\left({ }^{79} \mathrm{Br}\right)$. Moreover, enhancement-mode GaN transistors have been tested with $\gamma$-rays, heavy ions $\left({ }^{79} \mathrm{Br}\right.$ and $\left.{ }^{125} \mathrm{I}\right)$, and low-energy protons and have shown considerable hardness with respect to total dose and displacement damage.

\section{References}

[1] D. C. Look, D. C. Reynolds, J. W. Hemsky, J. R. Sizelove, R. L. Jones,and R. J. Molnar, Phys. Rev. Lett. 79, 22731997.

[2] L.D. Stevanovic et al., "Recent advances in silicon carbide MOSFET power devices", Proceedings of $25^{\text {st }}$ Applied Power Electronics Conference and exposition (APEC), pp. $401-407,2010$.

[3] W. Sung et al., "Design and investigation of frequency capability of $15 \mathrm{kV} 4 \mathrm{H}-\mathrm{SiC}$ IGBT", Proceedings of $21^{\text {st }}$ International Symposium on Power Semiconductor Devices \& ICs, pp. 271 274, 2009.

[4] H. Asai etr al., "Terrestrial Neutron-Induced Single-Event Burnout in SiC Power Diodes", IEEE Trans. Nucl. Sci., Vol. 59, Issue:4, Part:1, pp. 880 - 885, 2012.T.

[5] Makino et al., "Heavy-Ion Induced Anomalous Charge Collection From 4H-SiC Schottky Barrier Diodes”, IEEE Trans. Nucl. Sci., Vol. PP, issue: 99, 2013.

[6] S. Onoda et al., "Spatial, LET and range dependence of enhanced charge collection by single ion strike in 4H-SiC MESFETs", IEEE Trans. Nucl. Sci., Vol. 59, Issue:4, Part:1, pp. 742 - 748, 2012.

[7] M. Usman et al., "Impact of Ionizing Radiation on the $\mathrm{SiO} 2-\mathrm{SiC}$ Interface in $4 \mathrm{H}-\mathrm{SiC} \mathrm{BJTs}$ ", IEEE Trans. Nucl. Sci., Vol. 59, Issue: 12, pp. 3371 - 3376, 2012.

[8] A. Aktuk et al., "Radiation Effects in Commercial 1200V/24A Silicon Carbide Power MOSFETs", IEEE Trans. Nucl. Sci., Vol. 59, Issue:6, Part:2, pp. 3258 - 3264, 2012.

[9] G. Busatto et al., "Heavy-Ion Induced Single Event Gate Damage in Medium Voltage Power MOSFETs", IEEE Trans. Nucl. Sci., Vol. 56, no. 6, pp. 3573 - 3581, 2009.

[10] B. Weaver, P. A. Martin, J. B. Boos, C. Cress, "Displacement Damage Effects in AlGaN/GaN High Electron Mobility Transistors," Nuclear Science, IEEE Transactions on, vol. 59, pp. 3077-3080, 2012 